\title{
Invariant Submanifolds of Sasakian Manifolds Admitting Semisymmetric Nonmetric Connection
}

\author{
B. S. Anitha and C. S. Bagewadi \\ Department of Mathematics, Kuvempu University, Shankaraghatta, Karnataka, \\ Shimoga 577451, India \\ Correspondence should be addressed to C.S. Bagewadi, prof_bagewadi@yahoo.co.in
}

Received 30 March 2012; Accepted 2 July 2012

Academic Editor: V. R. Khalilov

Copyright (C) 2012 B. S. Anitha and C. S. Bagewadi. This is an open access article distributed under the Creative Commons Attribution License, which permits unrestricted use, distribution, and reproduction in any medium, provided the original work is properly cited.

\begin{abstract}
The object of this paper is to study invariant submanifolds $M$ of Sasakian manifolds $\widetilde{M}$ admitting a semisymmetric nonmetric connection, and it is shown that $M$ admits semisymmetric nonmetric connection. Further it is proved that the second fundamental forms $\sigma$ and $\bar{\sigma}$ with respect to Levi-Civita connection and semi-symmetric nonmetric connection coincide. It is shown that if the second fundamental form $\sigma$ is recurrent, 2-recurrent, generalized 2-recurrent, semiparallel, pseudoparallel, and Ricci-generalized pseudoparallel and $M$ has parallel third fundamental form with respect to semisymmetric nonmetric connection, then $M$ is totally geodesic with respect to Levi-Civita connection.
\end{abstract}

\section{Semisymmetric Nonmetric Connection}

The geometry of invariant submanifolds $M$ of Sasakian manifolds $\widetilde{M}$ is carried out from 1970's by M. Kon [1], D. Chinea [2], K. Yano and M. Kon [3] and B.S. Anitha and C.S. Bagewadi [4]. The aurthor [1] has proved that invariant submanifold of Sasakian structure also carries Sasakian structure. In this paper we extend the results to invariant submanifolds $M$ of Sasakian manifolds admitting Semisymmetric Nonmetric connection.

We know that a connection $\nabla$ on a manifold $M$ is called a metric connection if there is a Riemannian metric $g$ on $M$ if $\nabla g=0$; otherwise it is Nonmetric. Further it is said to be Semisymmetric if its torsion tensor $T(X, Y)=0$; thatis, $T(X, Y)=w(Y) X-w(X) Y$, where $w$ is a 1-form. A study of Semisymmetric connection on a Riemannian manifold was initiated by Yano [5]. In 1992, Agashe and Chafle [6] introduced the notion of Semisymmetric Nonmetric connection. If $\bar{\nabla}$ denotes Semisymmetric Nonmetric connection on a contact metric manifold, 
then it is given by [6]

$$
\bar{\nabla}_{X} Y=\nabla_{X} Y+\eta(Y) X
$$

where $\eta(Y)=g(Y, \xi)$.

The covariant differential of the $p$ th order, $p \geq 1$ of a $(0, k)$-tensor field $T, k \geq 1$ denoted by $\nabla^{p} T$, defined on a Riemannian manifold $(M, g)$ with the Levi-Civita connection $\nabla$. The tensor $T$ is said to be recurrent [7], if the following condition holds on $M$ :

$$
(\nabla T)\left(X_{1}, \ldots, X_{k} ; X\right) T\left(Y_{1}, \ldots, Y_{k}\right)=(\nabla T)\left(Y_{1}, \ldots, Y_{k} ; X\right) T\left(X_{1}, \ldots, X_{k}\right)
$$

respectively.

Consider

$$
\left(\nabla^{2} T\right)\left(X_{1}, \ldots, X_{k} ; X, Y\right) T\left(Y_{1}, \ldots, Y_{k}\right)=\left(\nabla^{2} T\right)\left(Y_{1}, \ldots, Y_{k} ; X, Y\right) T\left(X_{1}, \ldots, X_{k}\right)
$$

where $X, Y, X_{1}, Y_{1}, \ldots, X_{k}, Y_{k} \in T M$. From (1.2) it follows that at a point $x \in M$, if the tensor $T$ is nonzero, then there exists a unique 1 -form $\phi$, respectively, a $(0,2)$-tensor $\psi$, defined on a neighborhood $U$ of $x$ such that

$$
\nabla T=T \otimes \phi, \quad \phi=d(\log \|T\|),
$$

respectively.

The following

$$
\nabla^{2} T=T \otimes \psi
$$

holds on $U$, where $\|T\|$ denotes the norm of $T$ and $\|T\|^{2}=g(T, T)$. The tensor $T$ is said to be generalized 2-recurrent if

$$
\begin{aligned}
& \left(\left(\nabla^{2} T\right)\left(X_{1}, \ldots, X_{k} ; X, Y\right)-(\nabla T \otimes \phi)\left(X_{1}, \ldots, X_{k} ; X, Y\right)\right) T\left(Y_{1}, \ldots, Y_{k}\right) \\
& \quad=\left(\left(\nabla^{2} T\right)\left(Y_{1}, \ldots, Y_{k} ; X, Y\right)-(\nabla T \otimes \phi)\left(Y_{1}, \ldots, Y_{k} ; X, Y\right)\right) T\left(X_{1}, \ldots, X_{k}\right)
\end{aligned}
$$

holds on $M$, where $\phi$ is a 1 -form on $M$. From this it follows that at a point $x \in M$ if the tensor 
$T$ is nonzero, then there exists a unique $(0,2)$-tensor $\psi$, defined on a neighborhood $U$ of $x$, such that

$$
\nabla^{2} T=\nabla T \otimes \phi+T \otimes \psi
$$

holds on $U$.

\section{Isometric Immersion}

Let $f:(M, g) \rightarrow(\widetilde{M}, \widetilde{g})$ be an isometric immersion from an $n$-dimensional Riemannian manifold $(M, g)$ into $(n+d)$-dimensional Riemannian manifold $(\widetilde{M}, \widetilde{g}), n \geq 2, d \geq 1$. We denote $\nabla$ and $\widetilde{\nabla}$ as Levi-Civita connection of $M^{n}$ and $\widetilde{M}^{n+d}$, respectively. Then the formulas of Gauss and Weingarten are given by

$$
\begin{aligned}
& \tilde{\nabla}_{X} Y=\nabla_{X} Y+\sigma(X, Y), \\
& \tilde{\nabla}_{X} N=-A_{N} X+\nabla_{X}^{\perp} N,
\end{aligned}
$$

for any tangent vector fields $X, Y$ and the normal vector field $N$ on $M$, where $\sigma, A$, and $\nabla^{\perp}$ are the second fundamental form, the shape operator, and the normal connection, respectively. If the second fundamental form $\sigma$ is identically zero, then the manifold is said to be totally geodesic. The second fundamental form $\sigma$ and $A_{N}$ is related by

$$
\tilde{g}(\sigma(X, Y), N)=g\left(A_{N} X, Y\right)
$$

for tangent vector fields $X, Y$. The first and second covariant derivatives of the second fundamental form $\sigma$ are given by

$$
\begin{aligned}
\left(\tilde{\nabla}_{X} \sigma\right)(Y, Z)= & \nabla_{X}^{\perp}(\sigma(Y, Z))-\sigma\left(\nabla_{X} Y, Z\right)-\sigma\left(Y, \nabla_{X} Z\right), \\
\left(\tilde{\nabla}^{2} \sigma\right)(Z, W, X, Y)= & \left(\tilde{\nabla}_{X} \tilde{\nabla}_{Y} \sigma\right)(Z, W) \\
= & \nabla_{X}^{\perp}\left(\left(\tilde{\nabla}_{Y} \sigma\right)(Z, W)\right)-\left(\tilde{\nabla}_{Y} \sigma\right)\left(\nabla_{X} Z, W\right) \\
& -\left(\tilde{\nabla}_{X} \sigma\right)\left(Z, \nabla_{Y} W\right)-\left(\tilde{\nabla}_{\nabla_{X}} \sigma\right)(Z, W),
\end{aligned}
$$

respectively, where $\tilde{\nabla}$ is called the van der Waerden-Bortolotti connection of $M$ [8]. If $\tilde{\nabla} \sigma=0$, 
then $M$ is said to have parallel second fundamental form [8]. We next define endomorphisms $R(X, Y)$ and $X \wedge_{B} Y$ of $X(M)$ by

$$
\begin{gathered}
R(X, Y) Z=\nabla_{X} \nabla_{Y} Z-\nabla_{Y} \nabla_{X} Z-\nabla_{[X, Y]} Z \\
\left(X \wedge_{B} Y\right) Z=B(Y, Z) X-B(X, Z) Y
\end{gathered}
$$

respectively, where $X, Y, Z \in X(M)$ and $B$ is a symmetric $(0,2)$-tensor.

Now, for a $(0, k)$-tensor field $T, k \geq 1$ and a $(0,2)$-tensor field $B$ on $(M, g)$, we define the tensor $Q(B, T)$ by

$$
Q(B, T)\left(X_{1}, \ldots, X_{k} ; X, Y\right)=-\left(T\left(X \wedge_{B} Y\right) X_{1}, \ldots, X_{k}\right)-\cdots-T\left(X_{1}, \ldots, X_{k-1}\left(X \wedge_{B} Y\right) X_{k}\right)
$$

Putting into consideration the previous formula " $B=g, S$ and $T=\sigma$," we obtain the tensors $Q(g, \sigma)$ and $Q(S, \sigma)$.

\section{Sasakian Manifolds}

An $n$-dimensional differential manifold $M$ is said to have an almost contact structure $(\phi, \xi, \eta)$ if it carries a tensor field $\phi$ of type $(1,1)$, a vector field $\xi$, and 1 -form $\eta$ on $M$, respectively, such that

$$
\phi^{2}=-I+\eta \otimes \xi, \quad \eta(\xi)=1, \quad \eta \circ \phi=0, \quad \phi \xi=0 .
$$

Thus a manifold $M$ equipped with this structure is called an almost contact manifold and is denoted by $(M, \phi, \xi, \eta)$. If $g$ is a Riemannian metric on an almost contact manifold $M$ such that

$$
g(\phi X, \phi Y)=g(X, Y)-\eta(X) \eta(Y), \quad g(X, \xi)=\eta(X),
$$

where $X, Y$ are vector fields defined on $M$, then $M$ is said to have an almost contact metric structure $(\phi, \xi, \eta, g)$, and $M$ with this structure is called an almost contact metric manifold and is denoted by $(M, \phi, \xi, \eta, g)$.

If on $(M, \phi, \xi, \eta, g)$ the exterior derivative of 1-form $\eta$ satisfies

$$
\Phi(X, Y)=d \eta(X, Y)=g(X, \phi Y)
$$

then $(\phi, \xi, \eta, g)$ is said to be a contact metric structure and together with manifold $M$ is called contact metric manifold and $\Phi$ is a 2 -form. The contact metric structure $(M, \phi, \xi, \eta, g)$ is said to be normal if

$$
[\phi, \phi](X, Y)+2 d \eta \otimes \xi=0
$$


If the contact metric structure is normal, then it is called a Sasakian structure and $M$ is called a Sasakian manifold. Note that an almost contact metric manifold defines Sasakian structure if and only if

$$
\begin{gathered}
\left(\nabla_{X} \phi\right) Y=g(X, Y) \xi-\eta(Y) X \\
\nabla_{X} \xi=-\phi X .
\end{gathered}
$$

\section{Example of Sasakian Manifold}

Consider the 3-dimensional manifold $M=\left\{(x, y, z) \in R^{3}\right\}$, where $(x, y, z)$ are the standard coordinates in $R^{3}$. Let $\left\{E_{1}, E_{2}, E_{3}\right\}$ be linearly independent global frame field on $M$ given by

$$
E_{1}=\frac{\partial}{\partial x}-2 y \frac{\partial}{\partial z}, \quad E_{2}=\frac{\partial}{\partial y}, \quad E_{3}=\frac{\partial}{\partial z}
$$

Let $g$ be the Riemannian metric defined by

$$
\begin{aligned}
& g\left(E_{1}, E_{2}\right)=g\left(E_{1}, E_{3}\right)=g\left(E_{2}, E_{3}\right)=0, \\
& g\left(E_{1}, E_{1}\right)=g\left(E_{2}, E_{2}\right)=g\left(E_{3}, E_{3}\right)=1 .
\end{aligned}
$$

The $(\phi, \xi, \eta)$ is given by

$$
\begin{gathered}
\eta=2 y d x+d z, \quad \xi=E_{3}=\frac{\partial}{\partial z}, \\
\phi E_{1}=E_{2}, \quad \phi E_{2}=-E_{1}, \quad \phi E_{3}=0 .
\end{gathered}
$$

The linearity property of $\phi$ and $g$ yields

$$
\begin{gathered}
\eta\left(E_{3}\right)=1, \quad \phi^{2} U=-U+\eta(U) E_{3}, \\
g(\phi U, \phi W)=g(U, W)-\eta(U) \eta(W), \quad g(U, \xi)=\eta(U),
\end{gathered}
$$

for any vector fields $U, W$ on $M$. By definition of Lie bracket, we have

$$
\left[E_{1}, E_{2}\right]=2 E_{3}
$$

Let $\nabla$ be the Levi-Civita connection with respect to previously mentioned metric $g$ and be given by Koszula formula

$$
\begin{aligned}
2 g\left(\nabla_{X} Y, Z\right)= & X(g(Y, Z))+Y(g(Z, X))-Z(g(X, Y)) \\
& -g(X,[Y, Z])-g(Y,[X, Z])+g(Z,[X, Y])
\end{aligned}
$$


Then, we have

$$
\begin{array}{lcc}
\nabla_{E_{1}} E_{1}=0, & \nabla_{E_{1}} E_{2}=E_{3}, & \nabla_{E_{1}} E_{3}=-E_{2}, \\
\nabla_{E_{2}} E_{1}=-E_{3}, & \nabla_{E_{2}} E_{2}=0, & \nabla_{E_{2}} E_{3}=E_{1}, \\
\nabla_{E_{3}} E_{1}=-E_{2}, & \nabla_{E_{3}} E_{2}=E_{1}, & \nabla_{E_{3}} E_{3}=0 .
\end{array}
$$

The tangent vectors $X$ and $Y$ to $M$ are expressed as linear combination of $E_{1}, E_{2}, E_{3}$; that is, $X=a_{1} E_{1}+a_{2} E_{2}+a_{3} E_{3}$ and $Y=b_{1} E_{1}+b_{2} E_{2}+b_{3} E_{3}$, where $a_{i}$ and $b_{j}$ are scalars. Clearly $(\phi, \xi, \eta, g)$ and $X, Y$ satisfy (3.1), (3.2), (3.5), and (3.6). Thus $M$ is a Sasakian manifold. Further the following relations hold:

$$
\begin{gathered}
R(X, Y) Z=\{g(Y, Z) X-g(X, Z) Y\}, \\
R(X, Y) \xi=\{\eta(Y) X-\eta(X) Y\}, \\
R(\xi, X) Y=\{g(X, Y) \xi-\eta(Y) X\}, \\
R(\xi, X) \xi=\{\eta(X) \xi-X\}, \\
S(X, \xi)=(n-1) \eta(X), \\
Q \xi=(n-1) \xi,
\end{gathered}
$$

for all vector fields, $X, Y, Z$ and where $\nabla$ denotes the operator of covariant differentiation with respect to $g, \phi$ is a $(1,1)$ tensor field, $S$ is the Ricci tensor of type $(0,2)$, and $R$ is the Riemannian curvature tensor of the manifold.

\section{Invariant Submanifolds of Sasakian Manifolds Admitting Semisymmetric Nonmetric Connection}

If $\widetilde{M}$ is a Sasakian manifold with structure tensors $(\tilde{\phi}, \tilde{\xi}, \tilde{\eta}, \widetilde{g})$, then we know that its invariant submanifold $M$ has the induced Sasakian structure $(\phi, \xi, \eta, g)$.

A submanifold $M$ of a Sasakian manifold $\widetilde{M}$ with a Semisymmetric Nonmetric connection is called an invariant submanifold of $\widetilde{M}$ with a Semisymmetric Nonmetric connection, if for each $x \in M, \phi\left(T_{x} M\right) \subset T_{x} M$. As a consequence, $\xi$ becomes tangent to $M$. For an invariant submanifold of a Sasakian manifold with a Semisymmetric Nonmetric connection we have

$$
\sigma(X, \xi)=0
$$

for any vector $X$ tangent to $M$.

Let $\widetilde{M}$ be a Sasakian manifold admitting a Semisymmetric Nonmetric connection $\tilde{\nabla}$. 
Lemma 4.1. Let $M$ be an invariant submanifold of contact metric manifold $\widetilde{M}$ which admits Semisymmetric Nonmetric connection $\overline{\widetilde{\nabla}}$, and let $\sigma$ and $\bar{\sigma}$ be the second fundamental forms with respect to Levi-Civita connection and Semisymmetric Nonmetric connection; then (1) $M$ admits Semisymmetric Nonmetric connection and (2) the second fundamental forms with respect to $\widetilde{\nabla}$ and $\overline{\widetilde{\nabla}}$ are equal.

Proof. We know that the contact metric structure $(\tilde{\phi}, \tilde{\xi}, \tilde{\eta}, \widetilde{g})$ on $\widetilde{M}$ induces $(\phi, \xi, \eta, g)$ on invariant submanifold. By virtue of (1.1), we get

$$
\overline{\widetilde{\nabla}}_{X} Y=\tilde{\nabla}_{X} Y+\eta(Y) X
$$

By using (2.1) in (4.2), we get

$$
\overline{\widetilde{\nabla}}_{X} Y=\nabla_{X} Y+\sigma(X, Y)+\eta(Y) X
$$

Now Gauss formula (2.1) with respect to Semisymmetric Nonmetric connection is given by

$$
\overline{\widetilde{\nabla}}_{X} Y=\bar{\nabla}_{X} Y+\bar{\sigma}(X, Y)
$$

Equating (4.3) and (4.4), we get (1.1) and

$$
\bar{\sigma}(X, Y)=\sigma(X, Y)
$$

Now we introduce the definitions of semiparallel, pseudoparallel, and Riccigeneralized pseudoparallel with respect to Semisymmetric Nonmetric connection.

Definition 4.2. An immersion is said to be semiparallel, pseudoparallel, and Ricci-generalized pseudoparallel with respect to Semisymmetric Nonmetric connection, respectively, if the following conditions hold for all vector fields $X, Y$ tangent to $M$ :

$$
\begin{gathered}
\overline{\widetilde{R}} \cdot \sigma=0, \\
\overline{\widetilde{R}} \cdot \sigma=L_{1} Q(g, \sigma), \\
\overline{\widetilde{R}} \cdot \sigma=L_{2} Q(S, \sigma),
\end{gathered}
$$

where $\overline{\widetilde{R}}$ denotes the curvature tensor with respect to connection $\overline{\widetilde{\nabla}}$. Here $L_{1}$ and $L_{2}$ are functions depending on $\sigma$. 
Lemma 4.3. Let $M$ be an invariant submanifold of contact manifold $\widetilde{M}$ which admits Semisymmetric Nonmetric connection. Then Gauss and Weingarten formulae with respect to Semisymmetric Nonmetric connection are given by

$$
\begin{aligned}
\tan (\overline{\widetilde{R}}(X, Y) Z)= & R(X, Y) Z+\eta\left(\nabla_{Y} Z\right) X+\eta(Z) \nabla_{X} Y+\eta(Z) \eta(Y) X \\
& -\eta\left(\nabla_{X} Z\right) Y-\eta(Z) \nabla_{Y} X-\eta(Z) \eta(X) Y-\eta(Z)[X, Y] \\
& +\tan \left\{\overline{\tilde{\nabla}}_{X}\{\sigma(Y, Z)\}-\overline{\widetilde{\nabla}}_{Y}\{\sigma(X, Z)\}-\overline{\tilde{\nabla}}_{Y} \eta(Z) X+\overline{\widetilde{\nabla}}_{X} \eta(Z) Y\right\} \\
\operatorname{nor}(\overline{\tilde{R}}(X, Y) Z)= & \sigma\left(X, \nabla_{Y} Z\right)+\eta(Z) \sigma(X, Y)-\sigma\left(Y, \nabla_{X} Z\right)-\eta(Z) \sigma(Y, X)-\sigma([X, Y], Z) \\
& +\operatorname{nor}\left\{\tilde{\widetilde{\nabla}}_{X}\{\sigma(Y, Z)\}-\overline{\tilde{\nabla}}_{Y}\{\sigma(X, Z)\}-\overline{\tilde{\nabla}}_{Y} \eta(Z) X+\overline{\tilde{\nabla}}_{X} \eta(Z) Y\right\}
\end{aligned}
$$

Proof. The Riemannian curvature tensor $\widetilde{R}$ on $\widetilde{M}$ with respect to Semisymmetric Nonmetric connection is given by

$$
\overline{\widetilde{R}}(X, Y) Z=\overline{\widetilde{\nabla}}_{X} \overline{\widetilde{\nabla}}_{Y} Z-\overline{\widetilde{\nabla}}_{Y} \overline{\widetilde{\nabla}}_{X} Z-\overline{\widetilde{\nabla}}_{[X, Y]} Z
$$

Using (1.1) and (2.1) in (4.9), we get

$$
\begin{aligned}
\overline{\widetilde{R}}(X, Y) Z= & R(X, Y) Z+\sigma\left(X, \nabla_{Y} Z\right)+\eta\left(\nabla_{Y} Z\right) X+\overline{\widetilde{\nabla}}_{X}\{\sigma(Y, Z)\}+\overline{\widetilde{\nabla}}_{X} \eta(Z) Y \\
& +\eta(Z) \nabla_{X} Y+\eta(Z) \sigma(X, Y)+\eta(Z) \eta(Y) X-\sigma\left(Y, \nabla_{X} Z\right)-\eta\left(\nabla_{X} Z\right) Y \\
& -\overline{\widetilde{\nabla}}_{Y}\{\sigma(X, Z)\}-\overline{\tilde{\nabla}}_{Y} \eta(Z) X-\eta(Z) \nabla_{Y} X \\
& -\eta(Z) \sigma(Y, X)-\eta(Z) \eta(X) Y-\sigma([X, Y], Z)-\eta(Z)[X, Y] .
\end{aligned}
$$

Comparing tangential and normal part of (4.10), we obtain Gauss and Weingarten formulae (4.7) and (4.8).

Lemma 4.4. Let $M$ be an invariant submanifold of contact manifold $\widetilde{M}$ which admits Semisymmetric Nonmetric connection. If $\sigma$ is semiparallel, pseudoparallel, and Ricci-generalized pseudoparallel with 
respect to Semisymmetric Nonmetric connection, then we have

$$
\begin{aligned}
(\overline{\tilde{R}}(X, Y) \cdot \sigma)(U, V)= & R^{\perp}(X, Y) \sigma(U, V)-\sigma(R(X, Y) U, V)-\sigma(U, R(X, Y) V) \\
& -\nabla_{X} A_{\sigma(U, V)} Y+\nabla_{Y} A_{\sigma(U, V)} X-A_{\nabla_{Y}^{\perp} \sigma(U, V)} X+A_{\nabla_{X}^{\perp} \sigma(U, V)} Y \\
& +A_{\sigma(U, V)}[X, Y]-\sigma\left(X, A_{\sigma(U, V)} Y\right)+\sigma\left(Y, A_{\sigma(U, V)} X\right) \\
& -\eta\left(A_{\sigma(U, V)} Y\right) X+\eta\left(A_{\sigma(U, V)} X\right) Y-\eta\left(\nabla_{Y} U\right) \sigma(X, V) \\
& -\eta(U) \sigma\left(\nabla_{X} Y, V\right)-\eta(U) \eta(Y) \sigma(X, V)+\eta\left(\nabla_{X} U\right) \sigma(Y, V) \\
& +\eta(U) \sigma\left(\nabla_{Y} X, V\right)+\eta(U) \eta(X) \sigma(Y, V)+\eta(U) \sigma([X, Y], V) \\
& -\sigma\left(\overline{\tilde{\nabla}}_{X} \eta(U) Y, V\right)+\sigma\left(\overline{\tilde{\nabla}}_{Y} \eta(U) X, V\right)-\sigma\left(\overline{\tilde{\nabla}}_{X}\{\sigma(Y, U)\}, V\right) \\
& +\sigma\left(\overline{\tilde{\nabla}}_{Y}\{\sigma(X, U)\}, V\right)-\sigma\left(\sigma\left(X, \nabla_{Y} U\right), V\right)-\eta(U) \sigma(\sigma(X, Y), V) \\
& +\sigma\left(\sigma\left(Y, \nabla_{X} U\right), V\right)+\eta(U) \sigma(\sigma(Y, X), V)+\sigma(\sigma([X, Y], U), V) \\
& -\eta\left(\nabla_{Y} V\right) \sigma(U, X)-\eta(V) \sigma\left(U, \nabla_{X} Y\right)-\eta(V) \eta(Y) \sigma(U, X) \\
& +\eta\left(\nabla_{X} V\right) \sigma(U, Y)+\eta(V) \sigma\left(U, \nabla_{Y} X\right)+\eta(V) \eta(X) \sigma(U, Y) \\
& +\eta(V) \sigma(U,[X, Y])-\sigma\left(U, \overline{\tilde{\nabla}}_{X} \eta(V) Y\right)+\sigma\left(U, \bar{\nabla}_{Y} \eta(V) X\right) \\
& -\sigma\left(U, \overline{\widetilde{\nabla}}_{X}\{\sigma(Y, V)\}\right)+\sigma\left(U, \overline{\tilde{\nabla}}_{Y}\{\sigma(X, V)\}\right)-\sigma\left(U, \sigma\left(X, \nabla_{Y} V\right)\right) \\
& -\eta(V) \sigma(U, \sigma(X, Y))+\sigma\left(U, \sigma\left(Y, \nabla_{X} V\right)\right) \\
& +\eta(V) \sigma(U, \sigma(Y, X))+\sigma(U, \sigma([X, Y], V)) \\
& \\
& \\
&
\end{aligned}
$$

for all vector fields $X, Y, U$, and $V$ tangent to $M$, where

$$
R^{\perp}(X, Y)=\left[\nabla_{X}^{\perp}, \nabla_{Y}^{\perp}\right]-\nabla_{[X, Y]}^{\perp}
$$

Proof. We know, from tensor algebra, that

$$
(\overline{\widetilde{R}}(X, Y) \cdot \sigma)(U, V)=\overline{\widetilde{R}}(X, Y) \sigma(U, V)-\sigma(\overline{\widetilde{R}}(X, Y) U, V)-\sigma(U, \overline{\widetilde{R}}(X, Y) V) .
$$

Replacing $Z$ by $\sigma(U, V)$ in (4.9), we get

$$
\overline{\widetilde{R}}(X, Y) \sigma(U, V)=\overline{\widetilde{\nabla}}_{X} \overline{\tilde{\nabla}}_{Y} \sigma(U, V)-\overline{\widetilde{\nabla}}_{Y} \overline{\widetilde{\nabla}}_{X} \sigma(U, V)-\overline{\widetilde{\nabla}}_{[X, Y]} \sigma(U, V)
$$


In view of (1.1), (2.1), and (2.2), we have the following equalities:

$$
\begin{aligned}
\overline{\tilde{\nabla}}_{X} \overline{\tilde{\nabla}}_{Y} \sigma(U, V)= & \overline{\tilde{\nabla}}_{X}\left(-A_{\sigma(U, V)} Y+\nabla_{Y}^{\perp} \sigma(U, V)\right), \\
= & -\nabla_{X} A_{\sigma(U, V)} Y-\eta\left(A_{\sigma(U, V)} Y\right) X-\sigma\left(X, A_{\sigma(U, V)} Y\right) \\
& -A_{\nabla_{Y}^{\perp} \sigma(U, V)} X+\nabla_{X}^{\perp} \nabla_{Y}^{\perp} \sigma(U, V) .
\end{aligned}
$$

Similarly

$$
\begin{gathered}
\overline{\tilde{\nabla}}_{Y} \overline{\widetilde{\nabla}}_{X} \sigma(U, V) \\
=-\nabla_{Y} A_{\sigma(U, V)} X-\eta\left(A_{\sigma(U, V)} X\right) Y-\sigma\left(Y, A_{\sigma(U, V)} X\right)-A_{\nabla_{X}^{\perp} \sigma(U, V)} Y+\nabla_{Y}^{\perp} \nabla_{X}^{\perp} \sigma(U, V) \\
\overline{\widetilde{\nabla}}_{[X, Y]} \sigma(U, V)=-A_{\sigma(U, V)}[X, Y]+\nabla_{[X, Y]}^{\perp} \sigma(U, V) .
\end{gathered}
$$

Substituting (4.15), (4.16) and (4.17) into (4.14), we get

$$
\begin{aligned}
\overline{\widetilde{R}}(X, Y) \sigma(U, V)= & R^{\perp}(X, Y) \sigma(U, V)-\nabla_{X} A_{\sigma(U, V)} Y+\nabla_{Y} A_{\sigma(U, V)} X-A_{\nabla_{Y}^{\perp} \sigma(U, V)} X \\
& +A_{\nabla_{X}^{\perp} \sigma(U, V)} Y+A_{\sigma(U, V)}[X, Y]-\sigma\left(X, A_{\sigma(U, V)} Y\right)+\sigma\left(Y, A_{\sigma(U, V)} X\right) \\
& -\eta\left(A_{\sigma(U, V)} Y\right) X+\eta\left(A_{\sigma(U, V)} X\right) Y .
\end{aligned}
$$

By virtue of (4.10) in $\sigma(\overline{\widetilde{R}}(X, Y) U, V)$ and $\sigma(U, \overline{\widetilde{R}}(X, Y) V)$, we get

$$
\begin{aligned}
\sigma(\overline{\widetilde{R}}(X, Y) U, V)= & \sigma(R(X, Y) U, V)+\eta\left(\nabla_{Y} U\right) \sigma(X, V)+\eta(U) \sigma\left(\nabla_{X} Y, V\right) \\
& +\eta(U) \eta(Y) \sigma(X, V)-\eta\left(\nabla_{X} U\right) \sigma(Y, V)-\eta(U) \sigma\left(\nabla_{Y} X, V\right) \\
& -\eta(U) \eta(X) \sigma(Y, V)-\eta(U) \sigma([X, Y], V)+\sigma\left(\overline{\tilde{\nabla}}_{X} \eta(U) Y, V\right) \\
& -\sigma\left(\overline{\widetilde{\nabla}}_{Y} \eta(U) X, V\right)+\sigma\left(\overline{\widetilde{\nabla}}_{X}\{\sigma(Y, U)\}, V\right)-\sigma\left(\overline{\widetilde{\nabla}}_{Y}\{\sigma(X, U)\}, V\right) \\
& +\sigma\left(\sigma\left(X, \nabla_{Y} U\right), V\right)+\eta(U) \sigma(\sigma(X, Y), V)-\sigma\left(\sigma\left(Y, \nabla_{X} U\right), V\right) \\
& -\eta(U) \sigma(\sigma(Y, X), V)-\sigma(\sigma([X, Y], U), V),
\end{aligned}
$$




$$
\begin{aligned}
\sigma(U, \overline{\widetilde{R}}(X, Y) V)= & \sigma(U, R(X, Y) V)+\eta\left(\nabla_{Y} V\right) \sigma(U, X)+\eta(V) \sigma\left(U, \nabla_{X} Y\right) \\
& +\eta(V) \eta(Y) \sigma(U, X)-\eta\left(\nabla_{X} V\right) \sigma(U, Y)-\eta(V) \sigma\left(U, \nabla_{Y} X\right) \\
& -\eta(V) \eta(X) \sigma(U, Y)-\eta(V) \sigma(U,[X, Y])+\sigma\left(U, \overline{\widetilde{\nabla}}_{X} \eta(V) Y\right) \\
& -\sigma\left(U, \overline{\widetilde{\nabla}}_{Y} \eta(V) X\right)+\sigma\left(U, \overline{\widetilde{\nabla}}_{X}\{\sigma(Y, V)\}\right)-\sigma\left(U, \overline{\widetilde{\nabla}}_{Y}\{\sigma(X, V)\}\right) \\
& +\sigma\left(U, \sigma\left(X, \nabla_{Y} V\right)\right)+\eta(V) \sigma(U, \sigma(X, Y))-\sigma\left(U, \sigma\left(Y, \nabla_{X} V\right)\right) \\
& -\eta(V) \sigma(U, \sigma(Y, X))-\sigma(U, \sigma([X, Y], V)) .
\end{aligned}
$$

Substituting (4.18), (4.19) and (4.20) into (4.13), we get (4.11).

\section{Recurrent Invariant Submanifolds of Sasakian Manifolds Admitting Semisymmetric Nonmetric Connection}

We consider invariant submanifolds of a Sasakian manifold when $\sigma$ is recurrent, 2recurrent, and generalized 2-recurrent and $M$ has parallel third fundamental form with respect to Semisymmetric Nonmetric connection. We write (2.4) and (2.5) with respect to Semisymmetric Nonmetric connection, and they are given by

$$
\begin{aligned}
\left(\overline{\widetilde{\nabla}}_{X} \sigma\right)(Y, Z)= & \bar{\nabla}_{X}^{\perp}(\sigma(Y, Z))-\sigma\left(\bar{\nabla}_{X} Y, Z\right)-\sigma\left(Y, \bar{\nabla}_{X} Z\right), \\
\left(\overline{\widetilde{\nabla}}^{2} \sigma\right)(Z, W, X, Y)= & \left(\overline{\widetilde{\nabla}}_{X} \overline{\tilde{\nabla}}_{Y} \sigma\right)(Z, W) \\
= & \bar{\nabla}_{X}^{\perp}\left(\left(\overline{\widetilde{\nabla}}_{Y} \sigma\right)(Z, W)\right)-\left(\overline{\widetilde{\nabla}}_{Y} \sigma\right)\left(\bar{\nabla}_{X} Z, W\right) \\
& -\left(\overline{\tilde{\nabla}}_{X} \sigma\right)\left(Z, \bar{\nabla}_{Y} W\right)-\left(\overline{\tilde{\nabla}}_{\bar{\nabla}_{X} Y} \sigma\right)(Z, W) .
\end{aligned}
$$

We prove the following theorems.

Theorem 5.1. Let $M$ be an invariant submanifold of a Sasakian manifold $\widetilde{M}$ admitting a Semisymmetric Nonmetric connection. Then $\sigma$ is recurrent with respect to Semisymmetric Nonmetric connection if and only if it is totally geodesic with respect to Levi-Civita connection.

Proof. Let $\sigma$ be recurrent with respect to Semisymmetric Nonmetric connection; from (1.4) we get

$$
\left(\overline{\tilde{\nabla}}_{X} \sigma\right)(Y, Z)=\phi(X) \sigma(Y, Z)
$$


where $\phi$ is a 1 -form on $M$; in view of (5.1) and putting $Z=\xi$ in the above equation, we have

$$
\bar{\nabla}_{X}^{\perp} \sigma(Y, \xi)-\sigma\left(\bar{\nabla}_{X} Y, \xi\right)-\sigma\left(Y, \bar{\nabla}_{X} \xi\right)=\phi(X) \sigma(Y, \xi)
$$

By virtue of (4.1) in (5.4), we get

$$
-\sigma\left(\bar{\nabla}_{X} Y, \xi\right)-\sigma\left(Y, \bar{\nabla}_{X} \xi\right)=0
$$

Using (1.1), (3.1), (3.6), and (4.1) in (5.5), we get

$$
\sigma(Y, \phi X)-\sigma(Y, X)=0
$$

Replacing $X$ by $\phi X$ and by virtue of (3.1) and (4.1) in (5.6), we get

$$
-\sigma(Y, X)-\sigma(Y, \phi X)=0
$$

Adding (5.6) and (5.7), we obtain $\sigma(X, Y)=0$. Thus $M$ is totally geodesic. The converse statement is trivial. This proves the theorem.

Theorem 5.2. Let $M$ be an invariant submanifold of a Sasakian manifold $\widetilde{M}$ admitting a Semisymmetric Nonmetric connection. Then $M$ has parallel third fundamental form with respect to Semisymmetric Nonmetric connection if and only if it is totally geodesic with respect to Levi-Civita connection.

Proof. Let $M$ have parallel third fundamental form with respect to Semisymmetric Nonmetric connection. Then we have

$$
\left(\overline{\widetilde{\nabla}}_{X} \overline{\widetilde{\nabla}}_{Y \sigma}\right)(Z, W)=0
$$

Taking $W=\xi$ and using (5.2) in the above equation, we have

$$
\bar{\nabla}_{X}^{\perp}\left(\left(\overline{\widetilde{\nabla}}_{Y} \sigma\right)(Z, \xi)\right)-\left(\overline{\widetilde{\nabla}}_{Y \sigma}\right)\left(\bar{\nabla}_{X} Z, \xi\right)-\left(\overline{\widetilde{\nabla}}_{X} \sigma\right)\left(Z, \bar{\nabla}_{Y} \xi\right)-\left(\overline{\widetilde{\nabla}}_{\bar{\nabla}_{X} Y} \sigma\right)(Z, \xi)=0
$$

In view of (4.1) and by virtue of (5.1) in (5.9), we get

$$
\begin{aligned}
0= & -\bar{\nabla}_{X}^{\perp}\left\{\sigma\left(\bar{\nabla}_{Y} Z, \xi\right)+\sigma\left(Z, \bar{\nabla}_{Y} \xi\right)\right\}-\bar{\nabla}_{Y}^{\perp} \sigma\left(\bar{\nabla}_{X} Z, \xi\right)+\sigma\left(\bar{\nabla}_{Y} \bar{\nabla}_{X} Z, \xi\right) \\
& +2 \sigma\left(\bar{\nabla}_{X} Z, \bar{\nabla}_{Y} \xi\right)-\bar{\nabla}_{X}^{\perp} \sigma\left(Z, \bar{\nabla}_{Y} \xi\right)+\sigma\left(Z, \bar{\nabla}_{X} \bar{\nabla}_{Y} \xi\right)+\sigma\left(\bar{\nabla}_{\bar{\nabla}_{X} Y} Z, \xi\right)+\sigma\left(Z, \bar{\nabla}_{\bar{\nabla}_{X} Y} \xi\right) .
\end{aligned}
$$


Using (1.1), (3.1), (3.6), and (4.1) in (5.10), we get

$$
\begin{aligned}
0= & 2 \bar{\nabla}_{X}^{\perp} \sigma(Z, \phi Y)-2 \bar{\nabla}_{X}^{\perp} \sigma(Z, Y)-2 \eta(Z) \sigma(X, \phi Y)+2 \sigma\left(\nabla_{X} Z, Y\right) \\
& +2 \eta(Z) \sigma(X, Y)-\sigma\left(Z, \nabla_{X} \phi Y\right)-\sigma\left(Z, \phi \nabla_{X} Y\right)-\eta(Y) \sigma(Z, \phi X) \\
& +2 \sigma\left(Z, \nabla_{X} Y\right)+2 \eta(Y) \sigma(Z, X)-2 \sigma\left(\nabla_{X} Z, \phi Y\right) .
\end{aligned}
$$

Putting $Y=\xi$ and using (3.1), (3.6), and (4.1) in (5.11), we get

$$
0=\sigma(Z, X)-3 \sigma(Z, \phi X)
$$

Replacing $X$ by $\phi X$ and by virtue of (3.1) and (4.1) in (5.12), we get

$$
0=\sigma(Z, \phi X)+3 \sigma(Z, X)
$$

Multiplying (5.12) by 1 and (5.13) by 3 and adding these two equations, we obtain $\sigma(X, Z)=$ 0 . Thus $M$ is totally geodesic. The converse statement is trivial. This proves the theorem.

Corollary 5.3. Let $M$ be an invariant submanifold of a Sasakian manifold $\widetilde{M}$ admitting a Semisymmetric Nonmetric connection. Then $\sigma$ is 2-recurrent with respect to Semisymmetric Nonmetric connection if and only if it is totally geodesic with respect to Levi-Civita connection.

Proof. Let $\sigma$ be 2-recurrent with respect to Semisymmetric Nonmetric connection; from (1.5), we have

$$
\left(\overline{\widetilde{\nabla}}_{X} \overline{\widetilde{\nabla}}_{Y \sigma}\right)(Z, W)=\sigma(Z, W) \phi(X, Y)
$$

Taking $W=\xi$ and using (5.2) in the above equation, we have

$$
\begin{aligned}
& \bar{\nabla}_{X}^{\perp}\left(\left(\overline{\tilde{\nabla}}_{Y \sigma}\right)(Z, \xi)\right)-\left(\overline{\tilde{\nabla}}_{Y \sigma}\right)\left(\bar{\nabla}_{X} Z, \xi\right)-\left(\overline{\tilde{\nabla}}_{X} \sigma\right)\left(Z, \bar{\nabla}_{Y} \xi\right)-\left(\overline{\tilde{\nabla}}_{\bar{\nabla}_{X} Y} \sigma\right)(Z, \xi) \\
& \quad=\sigma(Z, \xi) \phi(X, Y) .
\end{aligned}
$$

In view of (4.1) and by virtue of (5.1) in (5.15), we get

$$
\begin{aligned}
0= & -\bar{\nabla}_{X}^{\perp}\left\{\sigma\left(\bar{\nabla}_{Y} Z, \xi\right)+\sigma\left(Z, \bar{\nabla}_{Y} \xi\right)\right\}-\bar{\nabla}_{Y}^{\perp} \sigma\left(\bar{\nabla}_{X} Z, \xi\right)+\sigma\left(\bar{\nabla}_{Y} \bar{\nabla}_{X} Z, \xi\right) \\
& +2 \sigma\left(\bar{\nabla}_{X} Z, \bar{\nabla}_{Y} \xi\right)-\bar{\nabla}_{X}^{\perp} \sigma\left(Z, \bar{\nabla}_{Y} \xi\right)+\sigma\left(Z, \bar{\nabla}_{X} \bar{\nabla}_{Y} \xi\right)+\sigma\left(\bar{\nabla}_{\bar{\nabla}_{X} Y} Z, \xi\right)+\sigma\left(Z, \bar{\nabla}_{\bar{\nabla}_{X} Y} \xi\right) .
\end{aligned}
$$


Using (1.1), (3.1), (3.6), and (4.1) in (5.16), we get

$$
\begin{aligned}
0= & 2 \bar{\nabla}_{X}^{\perp} \sigma(Z, \phi Y)-2 \bar{\nabla}_{X}^{\perp} \sigma(Z, Y)-2 \eta(Z) \sigma(X, \phi Y)+2 \sigma\left(\nabla_{X} Z, Y\right) \\
& +2 \eta(Z) \sigma(X, Y)-\sigma\left(Z, \nabla_{X} \phi Y\right)-\sigma\left(Z, \phi \nabla_{X} Y\right)-\eta(Y) \sigma(Z, \phi X) \\
& +2 \sigma\left(Z, \nabla_{X} Y\right)+2 \eta(Y) \sigma(Z, X)-2 \sigma\left(\nabla_{X} Z, \phi Y\right) .
\end{aligned}
$$

Putting $Y=\xi$ and using (3.1), (3.6), (4.1) in (5.17), we get

$$
0=\sigma(Z, X)-3 \sigma(Z, \phi X)
$$

Replacing $X$ by $\phi X$ and by virtue of (3.1) and (4.1) in (5.18), we get

$$
0=\sigma(Z, \phi X)+3 \sigma(Z, X)
$$

Multiplying (5.18) by 1 and (5.19) by 3 and adding these two equations, we obtain $\sigma(X, Z)=$ 0 . Thus $M$ is totally geodesic. The converse statement is trivial. This proves the theorem.

Theorem 5.4. Let $M$ be an invariant submanifold of a Sasakian manifold $\widetilde{M}$ admitting a Semisymmetric Nonmetric connection. Then $\sigma$ is generalized 2-recurrent with respect to Semisymmetric Nonmetric connection if and only if it is totally geodesic with respect to Levi-Civita connection.

Proof. Letting $\sigma$ be generalized 2-recurrent with respect to Semisymmetric Nonmetric connection, from (1.7), we have

$$
\left(\overline{\widetilde{\nabla}}_{X} \overline{\widetilde{\nabla}}_{Y \sigma}\right)(Z, W)=\psi(X, Y) \sigma(Z, W)+\phi(X)\left(\overline{\widetilde{\nabla}}_{Y} \sigma\right)(Z, W)
$$

where $\psi$ and $\phi$ are 2-recurrent and 1-form, respectively. Taking $W=\xi$ in (5.20) and using (4.1), we get

$$
\left(\overline{\widetilde{\nabla}}_{X} \overline{\widetilde{\nabla}}_{Y \sigma}\right)(Z, \xi)=\phi(X)\left(\overline{\tilde{\nabla}}_{Y \sigma}\right)(Z, \xi)
$$

Using (4.1) and (5.2) in above equation, we get

$$
\begin{aligned}
& \bar{\nabla}_{X}^{\perp}\left(\left(\overline{\widetilde{\nabla}}_{Y \sigma}\right)(Z, \xi)\right)-\left(\overline{\widetilde{\nabla}}_{Y \sigma}\right)\left(\bar{\nabla}_{X} Z, \xi\right)-\left(\overline{\widetilde{\nabla}}_{X} \sigma\right)\left(Z, \bar{\nabla}_{Y} \xi\right)-\left(\overline{\widetilde{\nabla}}_{\bar{\nabla}_{X} Y} \sigma\right)(Z, \xi) \\
& \quad=-\phi(X)\left\{\sigma\left(\bar{\nabla}_{Y} Z, \xi\right)+\sigma\left(Z, \bar{\nabla}_{Y} \xi\right)\right\} .
\end{aligned}
$$


In view of (4.1) and by virtue of (5.1) in (5.22), we get

$$
\begin{aligned}
& -\bar{\nabla}_{X}^{\perp}\left\{\sigma\left(\bar{\nabla}_{Y} Z, \xi\right)+\sigma\left(Z, \bar{\nabla}_{Y} \xi\right)\right\}-\bar{\nabla}_{Y}^{\perp} \sigma\left(\bar{\nabla}_{X} Z, \xi\right)+\sigma\left(\bar{\nabla}_{Y} \bar{\nabla}_{X} Z, \xi\right) \\
& +2 \sigma\left(\bar{\nabla}_{X} Z, \bar{\nabla}_{Y} \xi\right)-\bar{\nabla}_{X}^{\perp} \sigma\left(Z, \bar{\nabla}_{Y} \xi\right)+\sigma\left(Z, \bar{\nabla}_{X} \bar{\nabla}_{Y} \xi\right)+\sigma\left(\bar{\nabla}_{\bar{\nabla}_{X} Y} Z, \xi\right)+\sigma\left(Z, \bar{\nabla}_{\bar{\nabla}_{X} Y} \xi\right) \\
& =-\phi(X)\left\{\sigma\left(\bar{\nabla}_{Y} Z, \xi\right)+\sigma\left(Z, \bar{\nabla}_{Y} \xi\right)\right\} .
\end{aligned}
$$

Using (1.1), (3.1), (3.6), and (4.1) in (5.23), we get

$$
\begin{aligned}
0= & 2 \bar{\nabla}_{X}^{\perp} \sigma(Z, \phi Y)-2 \bar{\nabla}_{X}^{\perp} \sigma(Z, Y)-2 \eta(Z) \sigma(X, \phi Y)+2 \sigma\left(\nabla_{X} Z, Y\right)+2 \eta(Z) \sigma(X, Y) \\
& -\sigma\left(Z, \nabla_{X} \phi Y\right)-\sigma\left(Z, \phi \nabla_{X} Y\right)-\eta(Y) \sigma(Z, \phi X) \\
& +2 \sigma\left(Z, \nabla_{X} Y\right)+2 \eta(Y) \sigma(Z, X)-2 \sigma\left(\nabla_{X} Z, \phi Y\right) \\
= & -\phi(X)\{-\sigma(Z, \phi Y)+\sigma(Z, Y)\} .
\end{aligned}
$$

Putting $Y=\xi$ and using (3.1), (3.6), (4.1) in (5.24), we get

$$
0=\sigma(Z, X)-3 \sigma(Z, \phi X)
$$

Replacing $X$ by $\phi X$ and by virtue of (3.1) and (4.1) in (5.25), we get

$$
0=\sigma(Z, \phi X)+3 \sigma(Z, X)
$$

Multiplying (5.25) by 1 and (5.26) by 3 and adding these two equations, we obtain $\sigma(X, Z)=$ 0 . Thus $M$ is totally geodesic. The converse statement is trivial. This proves the theorem.

\section{Semiparallel, Pseudoparallel, and Ricci-Generalized Pseudoparallel Invariant Submanifolds of Sasakian Manifolds Admitting Semisymmetric Nonmetric Connection}

We consider invariant submanifolds of Sasakian manifolds admitting Semisymmetric Nonmetric connection satisfying the conditions $\overline{\widetilde{R}} \cdot \sigma=0, \overline{\widetilde{R}} \cdot \sigma=L_{1} Q(g, \sigma), \overline{\widetilde{R}} \cdot \sigma=L_{2} Q(S, \sigma)$.

Theorem 6.1. Let $M$ be an invariant submanifold of a Sasakian manifold $\widetilde{M}$ admitting a Semisymmetric Nonmetric connection. Then we prove that $M$ is semiparallel with respect to Semisymmetric Nonmetric connection if and only if $6=2 \bar{\phi}+\xi$. 
Proof. Let $M$ be semiparallel $\overline{\widetilde{R}} \cdot \sigma=0$. Putting $X=V=\xi$ and by virtue of (3.1), (3.6), and (4.1) in (4.11), we get

$$
\begin{aligned}
0= & -\sigma(U, R(\xi, Y) \xi)-\sigma\left(\overline{\widetilde{\nabla}}_{\xi} \eta(U) Y, \xi\right)+\sigma\left(\overline{\tilde{\nabla}}_{Y} \eta(U) \xi, \xi\right)-\sigma\left(\overline{\widetilde{\nabla}}_{\xi} \sigma(Y, U), \xi\right) \\
& -\sigma\left(U, \nabla_{\xi} Y\right)+\sigma\left(U, \nabla_{Y} \xi\right)+\sigma(U,[\xi, Y])-\sigma\left(U, \overline{\widetilde{\nabla}}_{\xi} Y\right)+\sigma\left(U, \overline{\widetilde{\nabla}}_{Y \xi}\right)+\sigma(U, Y) .
\end{aligned}
$$

Using (1.1), (2.1), (3.6), (3.15), (4.1), and (5.1) in (6.1), we get

$$
0=3 \sigma(U, Y)-\sigma\left(\overline{\widetilde{\nabla}}_{\xi} \eta(U) Y, \xi\right)-\sigma(U, \phi Y)-\sigma\left(U, \nabla_{\xi} Y\right)
$$

By definition $\sigma$ is a vector-valued covariant tensor, and so $\sigma(U, Y)$ is a vector. Therefore $\overline{\widetilde{\nabla}}_{\xi} \sigma(Y, U)$ is a vector, and hence by $(4.1)$, we have

$$
\sigma\left(\overline{\widetilde{\nabla}}_{\xi} \sigma(Y, U), \xi\right)=0
$$

Then from (6.2), we get

$$
3 \sigma(U, Y)=\bar{\phi} \sigma(U, Y)+\sigma\left(U, \nabla_{\xi} Y\right)
$$

Interchanging $Y$ and $U$ in (6.4), we get

$$
3 \sigma(Y, U)=\bar{\phi} \sigma(Y, U)+\sigma\left(U, \nabla_{\xi} Y\right)
$$

Adding these tow equations, (6.4) and (6.5), we get

$$
6=2 \bar{\phi}+\xi
$$

Theorem 6.2. Let $M$ be an invariant submanifold of a Sasakian manifold $\widetilde{M}$ admitting a Semisymmetric Nonmetric connection. Then we prove that $M$ is pseudoparallel with respect to Semisymmetric Nonmetric connection if and only if $L_{1}=\bar{\phi}+\xi / 2-3$.

Proof. Let $M$ be pseudoparallel $\overline{\widetilde{R}} \cdot \sigma=L_{1} Q(g, \sigma)$. Putting $X=V=\xi$ and by virtue of (3.1), (3.6), and (4.1) in (2.7), (4.11), we get

$$
\begin{gathered}
-\sigma(U, R(\xi, Y) \xi)-\sigma\left(\overline{\widetilde{\nabla}}_{\xi} \eta(U) Y, \xi\right)+\sigma\left(\overline{\widetilde{\nabla}}_{Y} \eta(U) \xi, \xi\right)-\sigma\left(\overline{\widetilde{\nabla}}_{\xi} \sigma(Y, U), \xi\right)-\sigma\left(U, \nabla_{\xi} Y\right) \\
+\sigma\left(U, \nabla_{Y} \xi\right)+\sigma(U,[\xi, Y])-\sigma\left(U, \overline{\widetilde{\nabla}}_{\xi} Y\right)+\sigma\left(U, \overline{\widetilde{\nabla}}_{Y \xi}\right)+\sigma(U, Y)=-L_{1} \sigma(U, Y) .
\end{gathered}
$$


International Journal of Mathematics and Mathematical Sciences

Using (1.1), (2.1), (3.6), (3.15), (4.1), and (5.1) in (6.7), we get

$$
3 \sigma(U, Y)-\sigma\left(\overline{\tilde{\nabla}}_{\xi} \eta(U) Y, \xi\right)-\sigma(U, \phi Y)-\sigma\left(U, \nabla_{\xi} Y\right)=-L_{1} \sigma(U, Y) .
$$

Now by using (6.3) in (6.8), we get

$$
\left(3+L_{1}\right) \sigma(U, Y)=\bar{\phi} \sigma(U, Y)+\sigma\left(U, \nabla_{\xi} Y\right)
$$

Interchanging $Y$ and $U$ in (6.9), we get

$$
\left(3+L_{1}\right) \sigma(Y, U)=\bar{\phi} \sigma(Y, U)+\sigma\left(Y, \nabla_{\xi} U\right)
$$

Adding (6.9) and (6.10), we get

$$
L_{1}=\bar{\phi}+\frac{\xi}{2}-3
$$

Theorem 6.3. Let $M$ be an invariant submanifold of a Sasakian manifold $\widetilde{M}$ admitting a Semisymmetric Nonmetric connection. Then we prove that $M$ is Ricci-generalized pseudoparallel with respect to Semisymmetric Nonmetric connection if and only if $L_{2}=(1 /(n-1))[\bar{\phi}+\xi / 2-3]$.

Proof. Let $M$ be Ricci-generalized pseudoparallel $\overline{\widetilde{R}} \cdot \sigma=L_{2} Q(S, \sigma)$. Putting $X=V=\xi$ and by virtue of (3.1), (3.6), (3.16), and (4.1) in (2.7), (4.11), we get

$$
\begin{aligned}
& -\sigma(U, R(\xi, Y) \xi)-\sigma\left(\overline{\tilde{\nabla}}_{\xi} \eta(U) Y, \xi\right)+\sigma\left(\overline{\widetilde{\nabla}}_{Y \eta}(U) \xi, \xi\right)-\sigma\left(\overline{\widetilde{\nabla}}_{\xi} \sigma(Y, U), \xi\right)-\sigma\left(U, \nabla_{\xi} Y\right) \\
& +\sigma\left(U, \nabla_{Y \xi}\right)+\sigma(U,[\xi, Y])-\sigma\left(U, \bar{\nabla}_{\xi} Y\right)+\sigma\left(U, \overline{\widetilde{\nabla}}_{Y \xi}\right)+\sigma(U, Y)=-L_{2}(n-1) \sigma(U, Y) .
\end{aligned}
$$

Using (1.1), (2.1), (3.6), (3.15), (4.1), and (5.1) in (6.12), we get

$$
3 \sigma(U, Y)-\sigma\left(\overline{\widetilde{\nabla}}_{\xi} \eta(U) Y, \xi\right)-\sigma(U, \phi Y)-\sigma\left(U, \nabla_{\xi} Y\right)=-L_{2}(n-1) \sigma(U, Y)
$$

Now by using (6.3) in (6.13), we get

$$
\left(3+L_{2}(n-1)\right) \sigma(U, Y)=\bar{\phi} \sigma(U, Y)+\sigma\left(U, \nabla_{\xi} Y\right)
$$

Interchanging $Y$ and $U$ in (6.14), we get

$$
\left(3+L_{2}(n-1)\right) \sigma(Y, U)=\bar{\phi} \sigma(Y, U)+\sigma\left(Y, \nabla_{\xi} U\right) .
$$


Adding (6.14) and (6.15), we get

$$
2\left(3+L_{2}(n-1)\right) \sigma(U, Y)=2 \bar{\phi} \sigma(U, Y)+\nabla_{\xi} \sigma(U, Y)
$$

Writting the above equation, we have

$$
L_{2}=\frac{1}{(n-1)}\left[\bar{\phi}+\frac{\xi}{2}-3\right]
$$

Remark 6.4. Let $M$ be an invariant submanifold of a Sasakian manifold which admits Semisymmetric Nonmetric connection. If $M$ is semiparallel, pseudoparallel, and Riccigeneralized pseudoparallel, then we have obtained conditions connecting $\phi, \xi, L_{1}$, and $L_{2}$. These conditions need further investigation and are to be interpreted geometrically.

Using Theorems 5.1 to 5.4 and corollary 5.3, we have the following result.

Corollary 6.5. Let $M$ be an invariant submanifold of a Sasakian manifold $\widetilde{M}$ admitting a Semisymmetric Nonmetric connection. Then the following statements are equivalent:

(1) ois recurrent,

(2) $\sigma$ is 2-recurrent,

(3) $\sigma$ is generalized 2-recurrent,

(4) $M$ has parallel third fundamental form.

\section{References}

[1] M. Kon, "Invariant submanifolds of normal contact metric manifolds," Kōdai Mathematical Seminar Reports, vol. 25, pp. 330-336, 1973.

[2] D. Chinea, "Invariant submanifolds of a quasi-K-Sasakian manifold," Rivista di Matematica della Università di Parma, vol. 4, no. 11, pp. 25-29, 1985.

[3] K. Yano and M. Kon, Structures on Manifolds, World Scientific, 1984.

[4] B. S. Anitha and C. S. Bagewadi, "Invariant submanifolds of Sasakian manifolds," communicated.

[5] K. Yano, "On semi-symmetric metric connection," Revue Roumaine de Mathématiques Pures et Appliquées, vol. 15, pp. 1579-1586, 1970.

[6] N. S. Agashe and M. R. Chafle, "A semi-symmetric nonmetric connection on a Riemannian manifold," Indian Journal of Pure and Applied Mathematics, vol. 23, no. 6, pp. 399-409, 1992.

[7] W. Roter, "On conformally recurrent Ricci-recurrent manifolds," Colloquium Mathematicum, vol. 46, no. 1, pp. 45-57, 1982.

[8] B.-Y. Chen, Geometry of Submanifolds and Its Applications, Science University of Tokyo, Tokyo, Japan, 1981. 


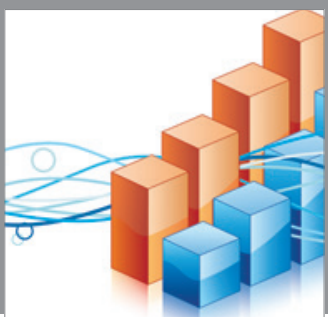

Advances in

Operations Research

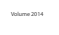

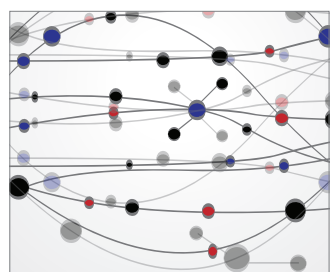

\section{The Scientific} World Journal
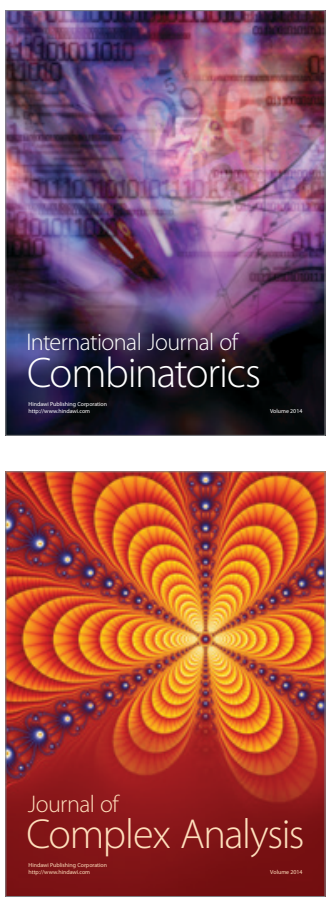

International Journal of

Mathematics and

Mathematical

Sciences
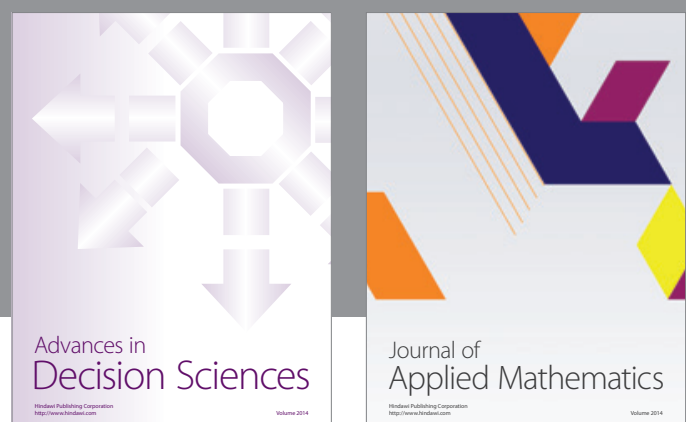

Journal of

Applied Mathematics
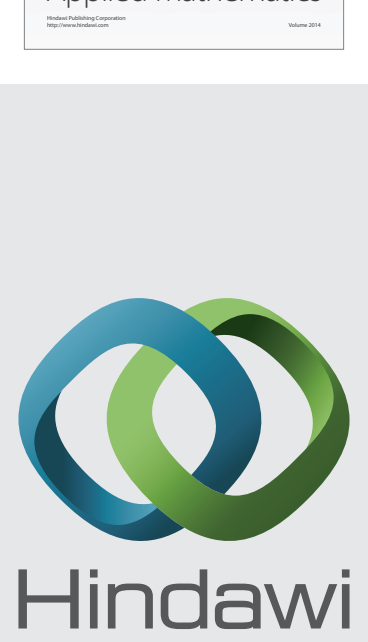

Submit your manuscripts at http://www.hindawi.com
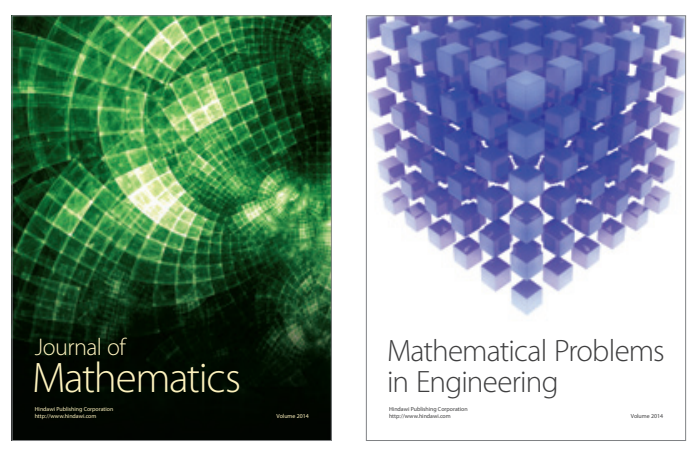

Mathematical Problems in Engineering
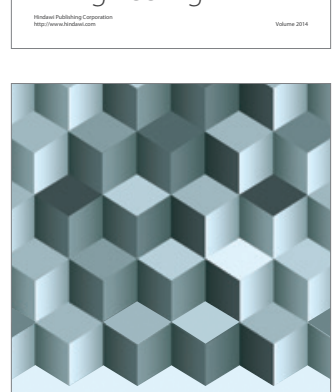

Journal of

Function Spaces
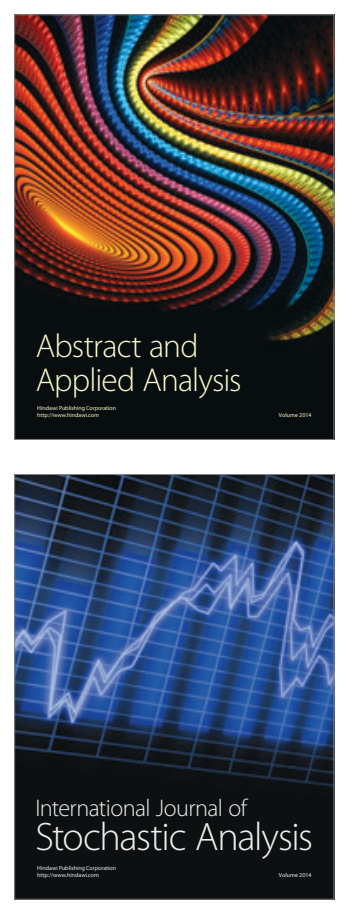

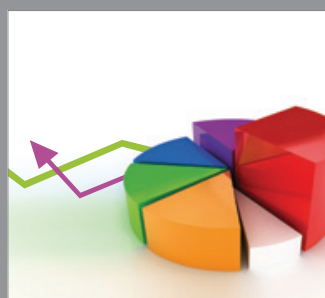

ournal of

Probability and Statistics

Promensencen
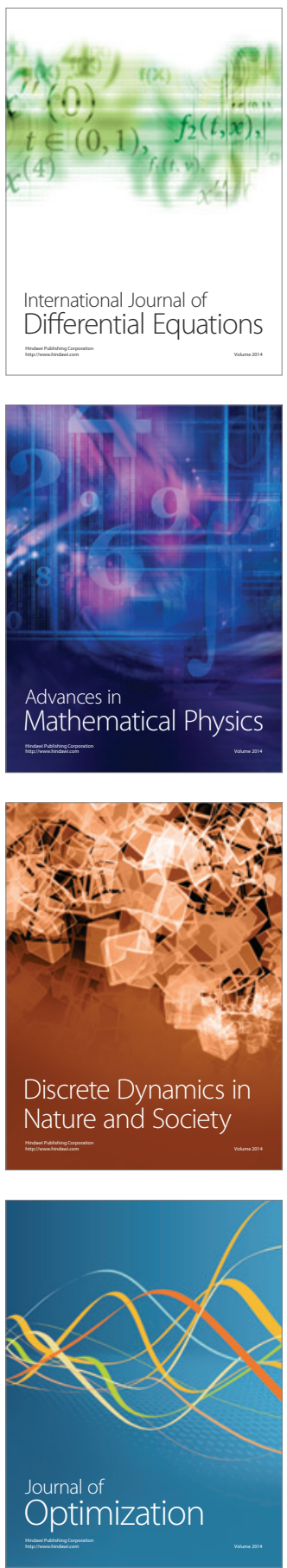